\title{
MULTIPLE TIDAL DISRUPTIONS AS AN INDICATOR OF BINARY SUPERMASSIVE BLACK HOLE SYSTEMS
}

\author{
Christopher Wegg and J. Nate Bode \\ Theoretical Astrophysics, California Institute of Technology, MC 350-17, 1200 East California Boulevard, \\ Pasadena, CA 91125, USA; wegg@tapir.caltech.edu \\ Received 2010 December 1; accepted 2011 July 18; published 2011 August 10
}

\begin{abstract}
We find that the majority of systems hosting multiple tidal disruptions (TDs) are likely to contain hard binary supermassive black hole (SMBH) systems, and also show that the rates of these repeated events are high enough to be detected by the Large Synoptic Survey Telescope (LSST) over its lifetime. Therefore, these multiple TD events provide a novel method for identifying SMBH binary systems with parsec to subparsec separations. The rates of TDs are investigated using simulations of non-interacting stars initially orbiting a primary SMBH and the potential of the model stellar cusp. The stars are then evolved forward in time and perturbed by a secondary SMBH inspiraling from the edge of the cusp to its stalling radius. We find with conservative magnitude estimates that the nextgeneration transient survey LSST should detect multiple TDs in approximately three galaxies over five years of observation, though less conservative estimates could increase this rate by an order of magnitude.
\end{abstract}

Key words: black hole physics - galaxies: evolution - galaxies: kinematics and dynamics - galaxies: nuclei

\section{INTRODUCTION}

Stars with radius $r_{\star}$ and mass $M_{\star}$, which pass within the tidal disruption radius $r_{t} \sim r_{\star}\left(M_{\mathrm{BH}} / M_{\star}\right)^{1 / 3}$ of a supermassive black hole (SMBH) of mass $M_{\mathrm{BH}}$, will be ripped apart by tidal forces. In the case of Sun-like stars,

$$
r_{t} \approx 1.2 r_{s} M_{8}^{-2 / 3}
$$

where $r_{s}$ is the Schwarzschild radius and $M_{8}$ is $M_{\mathrm{BH}} / 10^{8} M_{\odot}$. Therefore, when $M_{\mathrm{BH}} \gtrsim 10^{8} M_{\odot}$, the Schwarzschild radius lies outside $r_{t}$ and any Sun-sized star would be swallowed whole. Below this critical black hole mass, the star's debris is launched on orbits which span an energy range $\Delta E \approx G M_{\mathrm{BH}} r_{\star} / r_{t}^{2}$ (Rees 1988). This energy range is large compared to the energy of the highly elliptic initial orbit, and hence half the material will be initially unbound while half will begin to fall back onto the black hole. For main-sequence stars, the late-time canonical fall-back rate declines as $t^{-5 / 3}$ (Phinney 1989). This fall-back rate is initially super-Eddington for the canonical $10 \%$ accretion efficiency (Evans \& Kochanek 1989), but it is unclear whether a radiatively driven outflow results (Strubbe \& Quataert 2009; Lodato \& Rossi 2011) or the disk adjusts to lower its accretion rate.

Galaxies harboring an isolated SMBH at their center are expected to quickly clear a "loss cone" of orbits whose angular momenta about the black hole are low enough that their peribothra lie inside $r_{t}$. At this point, tidal disruptions (TDs) are predicted at a rate $\sim 10^{-4}-10^{-5} \mathrm{yr}^{-1}$ as stars diffuse into the loss cone (Magorrian \& Tremaine 1999; Wang \& Merritt 2004; Donley et al. 2002). The majority of candidate TDs thus far have been found through X-ray (e.g., Donley et al. 2002) or UV surveys (e.g., Gezari et al. 2008). This is expected, as can be seen by modeling the TD as a thick disk emitting as a blackbody with luminosity $L_{\text {edd }}$, temperature $T_{\text {eff }}$, and initially extending to $r_{t}$. In reality, the disk will expand outward on a viscous timescale and the initial super-Eddington rate could launch an outflow. Ignoring these complications, however, gives (Ulmer 1999)

$$
T_{\text {eff }} \sim 3.7 \times 10^{5} M_{8}^{1 / 12}\left(\frac{M_{\star}}{M_{\odot}}\right)^{-1 / 6}\left(\frac{r_{\star}}{r_{\odot}}\right)^{-1 / 2} \mathrm{~K},
$$

and the spectrum peaks in the extreme UV. Despite being in the Rayleigh-Jeans tail of this flux, optical transient surveys such as the Palomar Transient Factory (PTF), the Panoramic Survey Telescope and Rapid Response System (Pan-STARRS), and the Large Synoptic Survey Telescope (LSST) provide the prospect of finding many more TDs because of their unprecedented combination of high cadence and depth. It is expected that LSST will detect a striking $\sim 100-3000 \mathrm{yr}^{-1}$ (Strubbe \& Quataert 2009).

In this Letter, we calculate the rates of multiple TDs from a merging SMBH binary system and show that the detection of multiple TDs from a single galaxy likely indicates the galaxy hosts an SMBH binary with a parsec to subparsec separation. Our results are summarized in Table 1.

\section{SIMULATIONS}

We determine the rates of multiple TDs from a binary SMBH system using a simulation of $5 \times 10^{5}$ stars of radius $R_{\odot}$ orbiting the primary SMBH of mass $M_{1}$ in the test particle limit. The stars are initially chosen from a self-consistent isotropic stellar distribution centered on the primary SMBH (Tremaine et al. 1994; Dehnen 1993). Given its initial conditions, each star is then integrated forward in time whilst, at the same time, the secondary, of mass $q M_{1}$, is moved inward on a slightly eccentric inspiral until it reaches the stalling radius. During the integration, we ignore star-star interactions and, additionally, assume that the stellar potential does not change. If the star reaches $r_{t}$, it is counted as a TD, unless its angular momentum was initially within the TD loss cone.

Two mechanisms for enhanced rates of TDs in close SMBH binary systems have been considered in the literature: the Lidov-Kozai effect (Ivanov et al. 2005) and chaotic three-body orbits (Chen et al. 2009). We have extended these works by including two additional aspects: the evolution of the binary and the non-Keplerian stellar potential. ${ }^{1}$

\footnotetext{
During the review process, a further paper was published (Chen et al. 2011), which also considered the SMBH binary evolution and the stellar potential. Their disruption rates are consistent with ours.
} 
Table 1

Summary of Rates of Tidal Disruptions for Three Current and Upcoming Transient Surveys

\begin{tabular}{lccccccc}
\hline \hline Survey & $\begin{array}{c}\text { Magnitude Limit } \\
(g \text { band })\end{array}$ & $z_{\text {lim }}$ & $f_{\text {sky }}$ & $\mathcal{R}_{\text {single }}^{\text {(td) } / \mathrm{yr}^{-1}}$ & $\mathcal{R}_{\text {bin }}^{(\mathrm{td})} / \mathrm{yr}^{-1}$ & $N_{\text {single }}^{(\mathrm{multi})}$ & $N_{\text {bin }}^{(\mathrm{multi})}$ \\
\hline PTF & 21.0 & 0.06 & 0.2 & $0.7 \gamma$ & 0.02 & $0.9 \times 10^{-4} \gamma^{2}$ & 0.008 \\
Pan-STARRS (MDS) & 25.0 & 0.35 & $10^{-3}$ & $0.7 \gamma$ & 0.02 & $0.8 \times 10^{-4} \gamma^{2}$ & 0.007 \\
LSST & 25.0 & 0.35 & 0.5 & $300 \gamma$ & 8 & $0.03 \gamma^{2}$ \\
\hline
\end{tabular}

Notes. Symbols and calculation are described in the text. The numbers $N_{\text {single }}^{(\text {multi) }}$ and $N_{\text {bin }}^{(\text {multi) }}$ are for an observation time of $t_{\mathrm{obs}}=5 \mathrm{yr}$ and scale roughly as $t_{\mathrm{obs}}^{2}$.

The initial conditions of our stars are drawn from an $\eta$-model cusp with stellar mass $2 M_{1}$ (Tremaine et al. 1994; Dehnen 1993) whose density is given by

$$
\rho(r)=\frac{G M_{1} \eta}{2 \pi r_{c}^{3}} \frac{1}{\left(r / r_{c}\right)^{3-\eta}\left(1+r / r_{c}\right)^{1+\eta}} .
$$

Here, $r_{c}$ is the characteristic radius of the cusp and $\eta$ parameterizes the cusp steepness. The advantage of this model is that it self-consistently describes a finite mass of isotropic stars distributed around a central black hole together with their stellar potential. A centrally relaxed Bahcall-Wolf cluster corresponds to $\eta=1.25$. For the duration of the scattering experiments, the stellar potential is assumed to be centered on the primary SMBH and is not allowed to vary with time. These assumptions are made for simplicity, and will be relaxed in future studies.

For a given $\eta$ and SMBH mass ratio $q \leqslant 1$, our simulations depend only on the TD radius through $r_{t} / r_{c}$. The scaling of $r_{t} / r_{c}$ to real galaxies is described later by Equations (6) and (7).

The stars are initially on orbits, consistent with the primary SMBH and the stellar potential; however, their orbits are perturbed by the secondary SMBH. In a fully self-consistent simulation, the orbit of the secondary would evolve due to this exchange of energy with the stars. However, for efficiency and simplicity, the secondary SMBH's orbit follows an inspiral dominated by dynamical friction, halting at the stalling radius. Our approximation is checked in the lower panels of Figure 1, where we plot the change in stellar and binary energies. If the secondary path had been chosen perfectly, the two would lie on top of each other.

Specifically, the secondary SMBH is, at time $t=0$, given an eccentricity of 0.1 and an initial separation equal to the cusp radius, $r_{c}$. It is then migrated inward on a path governed by

$$
\frac{d \mathbf{v}}{d t}=-\frac{G\left[M_{1}(1+q)+M_{\star}(<r)\right]}{r^{3}} \mathbf{r}-\omega_{\mathrm{df}} \mathbf{v},
$$

where $M_{\star}(<r)$ is the stellar mass interior to $r$ and

$$
\omega_{\mathrm{df}}=\frac{4 \pi \log \Lambda q \mu \rho(<v)}{v^{3}}
$$

characterizes the dynamical friction (Binney \& Tremaine 2008). Here, $\rho(<v)$ is the density of stars at $r$ with velocity less than $v$. We have used a Coulomb $\log$ arithm that begins at $\log \Lambda \approx 4$, but which smoothly decreases to zero at the stalling radius calculated by Sesana et al. (2008). The functional form of the decrease was chosen to approximate the rate of shrinkage caused by the energy exchange with the stars during our simulations.

To perform our scattering experiments, we implemented the adaptive symplectic integrator described in Preto \& Tremaine (1999), with a time step which varies as $\Delta t \sim U^{-1}$, where $U$ is the potential energy. With this choice of time step, the integrator has the desirable property that it reproduces exactly Keplerian orbits independent of step size. Even for our mildly nonKeplerian orbits, this allows Kozai resonances to be correctly reproduced, since the spurious precession frequently found in other algorithms is absent. In addition, this integrator is well suited for this problem since it has been shown to correctly reproduce the highly eccentric orbits required for a star to be tidally disrupted (Peter 2009).

The results of our scattering experiments and the resultant disruption rates, $\Gamma_{\mathrm{td}}$, are shown in Figures 1 and 2 for $q=0.1$ and $q=0.3$, respectively. The plots are scaled by $\Omega \equiv$ $\left(2 G M_{1} / r_{c}^{3}\right)^{1 / 2}$.

The evolution of the rates can be understood qualitatively. Because we exclude stars initially in the loss cone, the rates are initially low. But, as the secondary inspirals, it enters denser regions and interacts with increasing numbers of stars, with a concomitant increase in the TD rate. The rate increases until the secondary reaches the stalling radius, where it clears a path and the rate begins to decline.

We find that the majority of TDs are due to the type of chaotic orbits described by Chen et al. (2009) as opposed to the Kozai effect discussed by Ivanov et al. (2005). This is demonstrated in the left-hand panel of Figure 3. The primary reason for this is that apsidal precession in the non-Keplerian potential destroys the secular Kozai effect for the majority of orbits.

Our rates are lower than those discussed by Chen et al. (2009) largely because we have considered less steep cusps. This both reduces the number of stars that can be disrupted as the binary hardens and increases the orbital timescale at the hardening radius. Both effects reduce the rate of disruptions. In addition, we have considered the binary evolution, which Chen et al. (2009) did not, although this has a smaller effect.

To apply our simulations to physical galaxies, we use the fits from Merritt et al. (2009) to the inner regions of Advanced Camera for Surveys (ACS) Virgo Cluster galaxies (Côté et al. 2004). For power-law galaxies, these give ${ }^{2}$

$$
r_{c}=22\left(M_{1} / 10^{8} M_{\odot}\right)^{0.55} \mathrm{pc} .
$$

We assume the stars have radius $R_{\odot}$, thus giving

$$
r_{t} / r_{c}=4.9 \times 10^{-7}\left(M_{1} / 10^{8} M_{\odot}\right)^{0.22}
$$

With these scalings, our simulations for $\eta=1.25$ and $q=0.3$ are shown in Figure 3.

\footnotetext{
2 D. Merritt (2010, private communication). From fitting to Figure 2 of
} Merritt et al. (2009). 


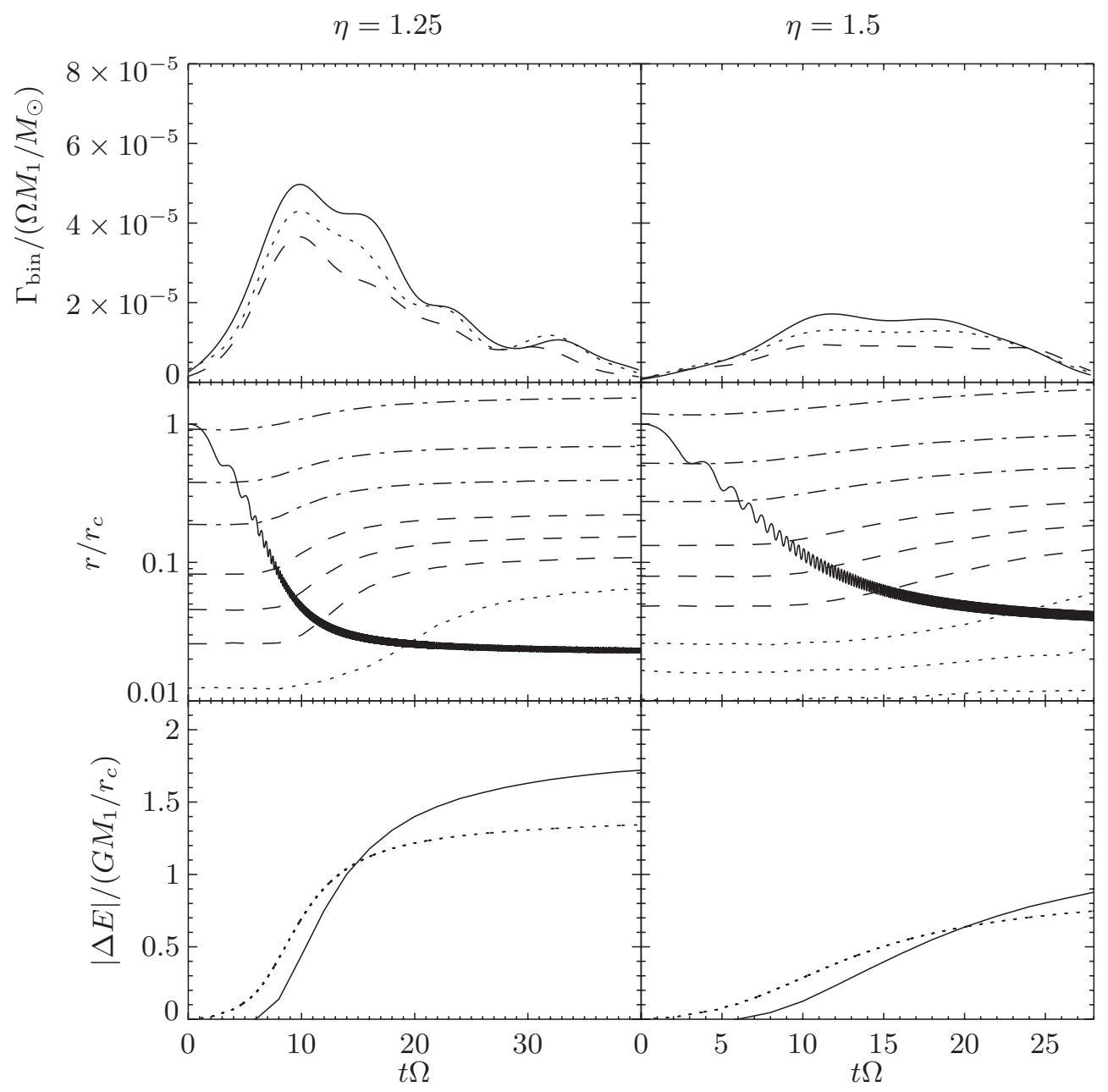

Figure 1. Results for mass ratio $q=0.1$. The left-hand panels show an $\eta=1.25$, Bahcall-Wolf cusp and the right-hand panels an $\eta=1.5$ cusp. The upper panel shows TD rate, $\Gamma_{\text {bin }}$, for TD to cusp radius ratios $r_{t} / r_{c}=(9,7,5) \times 10^{-7}$ in solid, dotted, and dashed lines, respectively. The distribution of disruption times has been kernel smoothed with a Gaussian of width $\sigma=2$. The middle panel shows the evolution of the binary separation by a solid line and radii enclosing $0.1 \%, 0.2 \%$, and $0.4 \%$ of the stellar mass by dotted, $1 \%, 2 \%$, and $4 \%$ by dashed, and $10 \%, 20 \%$, and $40 \%$ by dash-dotted lines. The lower panel shows the evolution of the energy of the binary by the solid line and the stars by the dotted line. In a fully self-consistent evolution, these would lie on top of each other. The simulations are scaled by $\Omega \equiv\left(2 G M_{1} / r_{c}^{3}\right)^{1 / 2}$.

\section{OBSERVABLE TIDAL DISRUPTIONS}

The absolute magnitude of an individual disruption has considerable modeling uncertainties and is likely to depend on many quantities such as the SMBH mass, the SMBH spin, and the geometry of the disruption (Strubbe \& Quataert 2009).

We instead derive a simple empirical estimate of the volume accessible by comparison to Gezari et al. (2008). Two luminous optical events coincident with UV flares were discovered in $\sim 2.9 \mathrm{deg}^{2}$. Their spectra and light curves were consistent with TD events, making this their most likely explanation. Their redshifts were $z=0.33$ and $z=0.37$, giving extinctioncorrected (but not $K$-corrected) absolute $g$-band magnitudes of -17.7 and -18.9 (Gezari 2010). Requiring that these two cases be 2 mag brighter than the $25.0 \mathrm{~g}$-band limit of LSST gives maximum redshifts of detection of $z=0.27$ and $z=0.43$, respectively. The 2 mag buffer better ensures a convincing light curve, which would display the characteristic fast rise and decay of a TD. Based on these numbers, we choose $z=0.35$ as the limit for LSST.

There is also only a small range of SMBH masses which needs to be considered. Because SMBHs of mass greater than $10^{8} M_{\odot}$ cannot tidally disrupt stars (Equation (1), ignoring SMBH spin), and SMBHs of mass less than $10^{7} M_{\odot}$ are significantly less luminous, (particularly if super-Eddington outflows are neglected), we restrict our analysis to SMBHs with masses between $10^{7}$ and $10^{8} M_{\odot}$.

\section{RATES OF SINGLE TIDAL DISRUPTIONS}

We now calculate the rate of TDs observable by LSST both for systems with isolated SMBHs and systems with SMBH binaries.

In the case of isolated SMBHs, the rate of TDs observed by LSST will be

$$
\mathcal{R}_{\text {single }}^{\mathrm{tdd})}=f_{\text {sky }} \int \frac{d N}{d M_{\mathrm{BH}}} V_{\mathrm{c}}\left(M_{\mathrm{BH}}\right) \Gamma_{\mathrm{td}}\left(M_{\mathrm{BH}}\right) d M_{\mathrm{BH}},
$$

where $f_{\text {sky }}$ is the fraction of the sky covered by LSST, $\Gamma_{\mathrm{td}}$ is the rate of TDs per galaxy, $V_{c}$ is the total co-moving volume over which a TD is observable, and $d N / d M_{\mathrm{BH}}$ is the black hole mass function. We have used the black hole mass function (Aller \& Richstone 2002)

$$
\frac{d N}{d M_{\mathrm{BH}}}=c\left(\frac{M_{\mathrm{BH}}}{M_{\mathrm{BH}}^{\star}}\right)^{-\alpha} e^{-M_{\mathrm{BH}} / M_{\mathrm{BH}}^{\star}},
$$




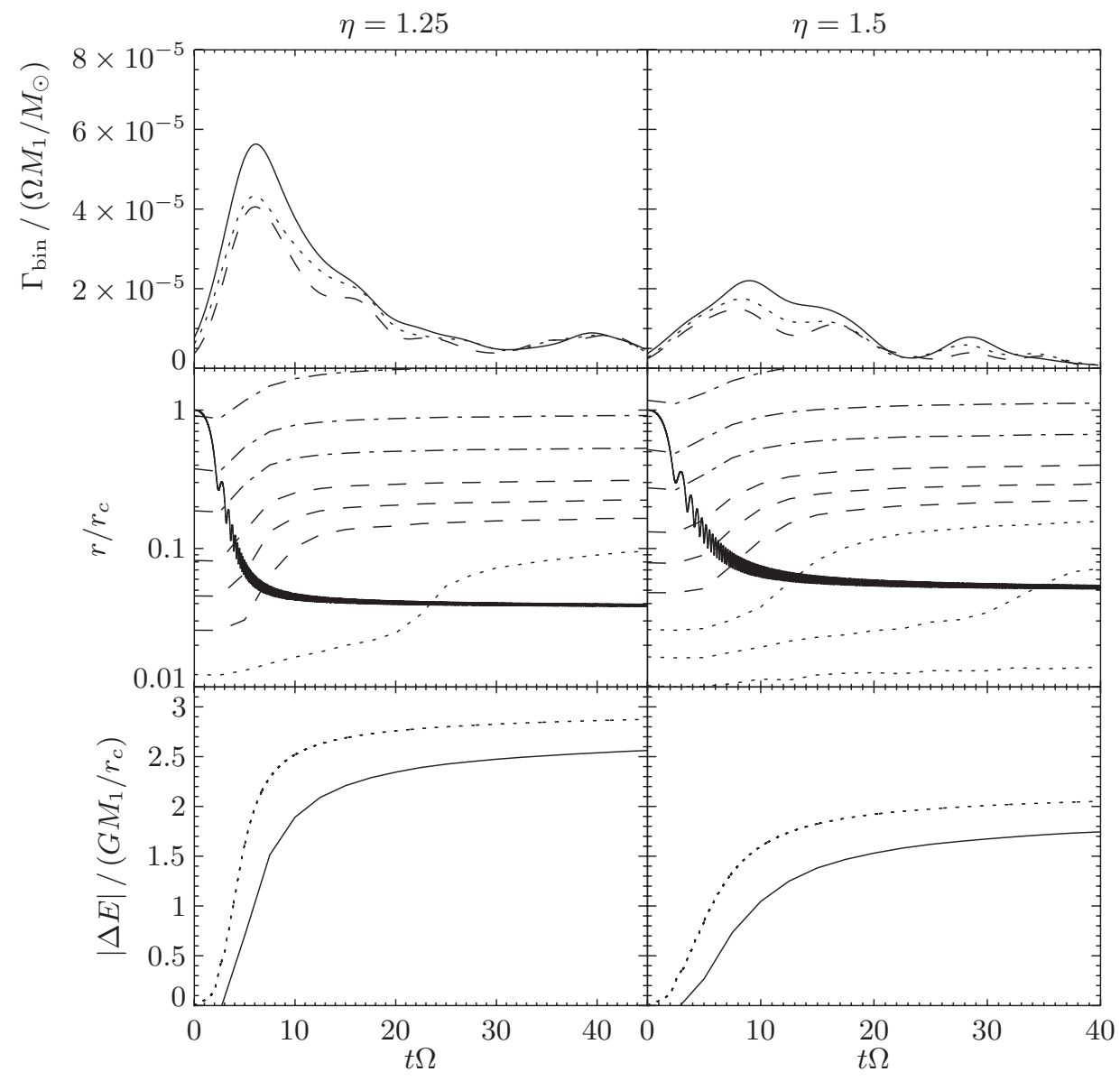

Figure 2. Results as described in Figure 1 for $q=0.3$.
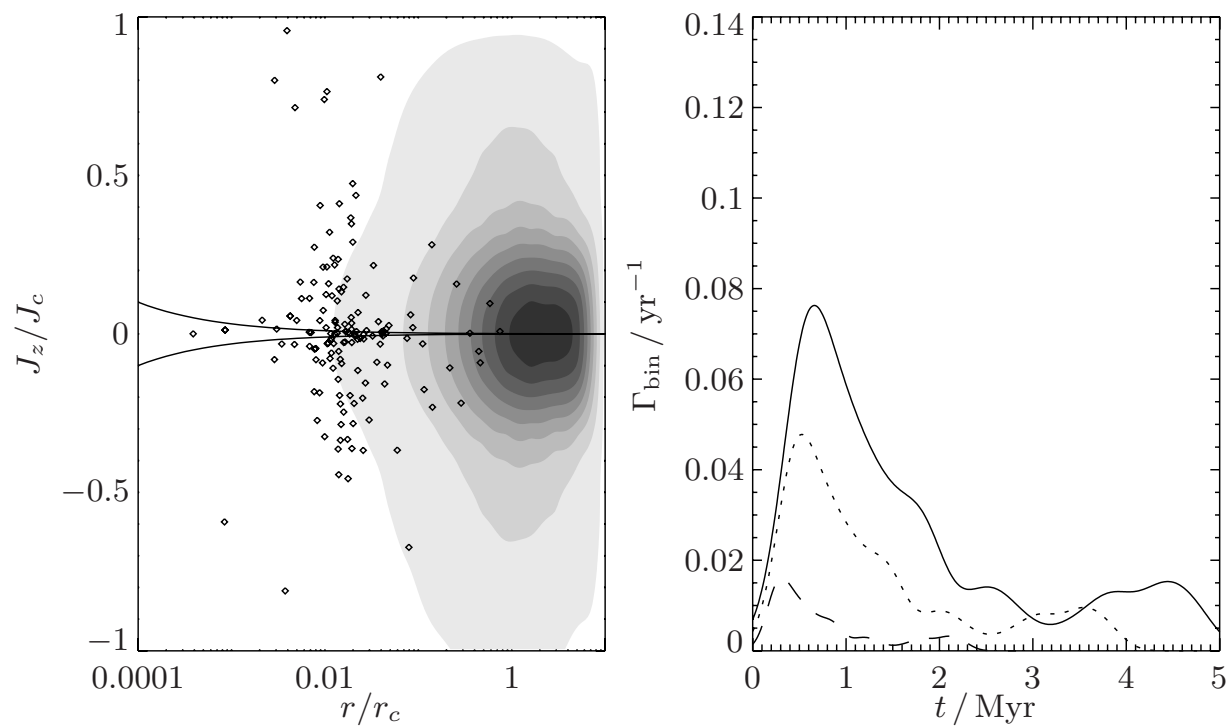

Figure 3. Plots of our $\eta=1.25, q=0.3$ simulation. Left panel shows the stars that are tidally disrupted for $r_{t} / r_{c}=5 \times 10^{-7}$ as a function of their initial radius and $z$-component of angular momentum normalized to the circular angular momentum at that radius, $J_{z} / J_{c}$. The Kozai wedge is plotted together with the overall stellar density. A large fraction of the disrupted stars lie well outside the Kozai wedge, indicating that these are chaotic orbits (see Chen et al. 2009). The contours show the initial stellar distribution, each is evenly spaced in density. The right-hand panel shows the rates from the same simulation scaled using the relations in Equations (6) and (7) for $M_{1}=10^{8} M_{\odot}$ by solid, $5 \times 10^{7} M_{\odot}$ by dotted, and $10^{7} M_{\odot}$ by dashed lines.

with the parameters $c=3 \times 10^{-11} M_{\odot}^{-1} \mathrm{Mpc}^{-3}, M_{\mathrm{BH}}^{\star}=$ $1.1 \times 10^{8} M_{\odot}$, and $\alpha=0.95$ (values derived by Aller \& Richstone 2002, scaled to $H_{0}=71 \mathrm{~km} \mathrm{~s}^{-1} \mathrm{Mpc}^{-1}$ ). The rate of TDs per galaxy is highly uncertain, and so we parameterize,
$\Gamma_{\mathrm{td}}=\gamma \times 10^{-5} \mathrm{yr}^{-1}$, scaling to the observationally motivated constant rate per galaxy of Donley et al. (2002) independent of $M_{\mathrm{BH}}$. We also assume that $V_{\mathrm{c}}$ is independent of $M_{\mathrm{BH}}$ and is $10.7 \mathrm{Gpc}^{3}$, corresponding to our redshift limit of $z=0.35$, 
with the assumption that $H_{0}=71 \mathrm{~km} \mathrm{~s}^{-1} \mathrm{Mpc}^{-1}$. Using these approximations and $f_{\text {sky }} \approx 0.5$, the rate of TDs detected by LSST in galaxies containing isolated SMBHs is predicted to be

$$
\begin{aligned}
\mathcal{R}_{\text {single }}^{\mathrm{tdd})} & \sim \Gamma_{\mathrm{td}} V_{\mathrm{c}} f_{\text {sky }} \int_{10^{7} M_{\odot}}^{10^{8} M_{\odot}} \frac{d N}{d M_{\mathrm{BH}}} d M_{\mathrm{BH}} \\
& =\Gamma_{\mathrm{td}} \times 3 \times 10^{7} \text { galaxies }=300 \gamma \mathrm{yr}^{-1} .
\end{aligned}
$$

Now consider systems hosting binary SMBHs. The rate of disruptions will be

$$
\mathcal{R}_{\text {bin }}^{\text {(td) }}=f_{\text {sky }} \int V_{\mathrm{c}} \Gamma_{\text {bin }}\left(M_{1}, q, t\right) R_{\text {merge }}\left(M_{1}, q\right) d q d M_{1} d t,
$$

where $R_{\text {merge }}\left(M_{1}, q\right) d q d M_{1}$ is the rate of mergers per unit comoving volume for binary SMBHs with primary mass between $M_{1}$ and $M_{1}+d M_{1}$ and with mass ratio between $q$ and $q+d q$. The quantity $\int \Gamma_{\text {bin }}\left(M_{1}, q, t\right) d t$ is the total number of TDs in a merger and was linearly interpolated/extrapolated from the simulations in Figures 1 and 2 together with Equations (6) and (7).

Over the narrow range of redshift and primary mass accessible, we approximate

$$
R_{\text {merge }}\left(M_{1}, q\right)=C \frac{d N}{d M_{1}} F(q)
$$

where we have assumed that the mass ratio distribution of $\mathrm{SMBH}$ binaries follows the local galaxy merger mass ratio distribution given by Stewart et al. (2009), $F(q)=q^{-0.25}(1-$ $q)^{1.1}$. The normalization constant $C=0.03 \mathrm{Gyr}^{-1}$ was chosen to reproduce the simulated local merger rate ${ }^{3}$ of $\mathrm{SMBH}$ binaries, with $10^{7} M_{\odot}<M_{1}<10^{8} M_{\odot}$ and $q>0.05$, of approximately $9 \times 10^{-5} \mathrm{Mpc}^{-3} \mathrm{Gyr}^{-1}$.

Using these approximations, then, if all galaxies have an $\eta=1.25$ cusp, we can expect LSST to detect

$$
\begin{aligned}
\mathcal{R}_{\text {bin }}^{(\mathrm{td})} \sim & V_{\mathrm{c}} f_{\text {sky }} C \int_{10^{7} M_{\odot}}^{10^{8} M_{\odot}} d M_{1} \int_{0.05}^{0.5} d q \\
& \times \int d t \frac{d N}{d M_{1}} F(q) \Gamma_{\text {bin }}\left(M_{1}, q, t\right)=8 \mathrm{yr}^{-1},
\end{aligned}
$$

where we have limited the mass ratio to $q<0.5$, since this is the limit of our simulations.

Note that the number of galaxies in the enhanced state which occurs for $\sim 10 / \Omega \sim 1.5\left(M_{1} / 10^{8} M_{\odot}\right)^{0.33} \mathrm{Myr}$ is

$$
\begin{aligned}
N_{\mathrm{enh}} \sim & V_{\mathrm{c}} f_{\text {sky }} C \int_{10^{7} M_{\odot}}^{10^{8} M_{\odot}} d M_{1} \int_{0.05}^{0.5} d q \\
& \times\left[\frac{d N}{d M_{1}} F(q) \times 10 / \Omega\left(M_{1}\right)\right]=400 .
\end{aligned}
$$

\section{RATES OF MULTIPLE TIDAL DISRUPTIONS}

We now calculate the rate of multiple TDs in systems containing both isolated and binary SMBHs.

In the case of isolated SMBHs, over a period of observing $t_{\mathrm{obs}}$ the total number of TDs will follow a Poisson distribution

\footnotetext{
3 M. Volonteri (2010, private communication). From data in Figure 2 of
} Volonteri et al. (2009). with mean $x \equiv t_{\mathrm{obs}} \Gamma_{\mathrm{td}}$. The probability of observing multiple TDs from a single galaxy is therefore $P(x)=1-e^{-x}-x e^{-x}$.

Then the expected number of isolated SMBHs exhibiting multiple TDs during $t_{\mathrm{obs}}$ is

$$
\begin{aligned}
N_{\text {single }}^{(\text {multi) }}= & V_{\mathrm{c}} f_{\text {sky }} \int_{10^{7} M_{\odot}}^{10^{8} M_{\odot}} \frac{d N}{d M_{\mathrm{BH}}} P\left(t_{\mathrm{obs}} \Gamma_{\mathrm{td}}\right) d M_{\mathrm{BH}} \\
& \sim 0.03 \gamma^{2}\left(t_{\mathrm{obs}} / 5 \mathrm{yr}\right)^{2}
\end{aligned}
$$

where in the final relation we have expanded in the small parameter $x \equiv t_{\mathrm{obs}} \Gamma_{\mathrm{td}}$.

Similarly, the expected number of multiple TDs observed from binary SMBHs is

$$
N_{\text {bin }}^{\text {(multi) }}=f_{\text {sky }} V_{\mathrm{c}} \int P\left(\Gamma_{\text {bin }} t_{\text {obs }}\right) R_{\text {merge }} d M_{1} d t d q .
$$

Using the same approximations as used in estimating Equation (13), we find over an observation time, $t_{\mathrm{obs}}=5 \mathrm{yr}$, the expected number of close binary SMBHs exhibiting multiple TDs observable by LSST to be

$$
\begin{aligned}
N_{\text {bin }}^{(\text {multi })} \sim & V_{\mathrm{c}} f_{\text {sky }} C \int_{10^{7} M_{\odot}}^{10^{8} M_{\odot}} d M_{1} \int_{0.05}^{0.5} d q \int d t \frac{d N}{d M_{\mathrm{BH}}} F(q) \\
& \times P\left(\Gamma_{\mathrm{bin}}\left(M_{1}, q, t\right) t_{\mathrm{obs}}\right)=3
\end{aligned}
$$

where $P\left(\Gamma_{\text {bin }} t_{\text {obs }}\right)$ was calculated from our simulations together with Equations (6) and (7).

Toward the upper end of the range $10^{7}-10^{8} M_{\odot}, \Gamma_{\text {bin }} t_{\text {obs }} \sim$ 0.5 for major mergers. This indicates that the majority of close SMBH binaries with primaries in the upper end of this range could potentially be identified using multiple disruptions. Equation (17) broadly scales as $\left(t_{\mathrm{obs}} / 5 \mathrm{yr}\right)^{2}$, but this is only approximate because $x \equiv \Gamma_{\text {bin }} t_{\mathrm{obs}} \sim 0.5$ for some systems.

\section{DISCUSSION}

We have estimated the enhanced rate of TDs from SMBH binaries and shown that if a system exhibiting multiple TDs is observed then, in our fiducial model, it is $\sim 100 / \gamma^{2}$ times more likely to be a close SMBH binary than an isolated SMBH system. It has also been shown that the upcoming transient survey LSST is likely to detect several systems with multiple disruptions during a five-year observation period.

Once a double TD is detected, these galaxies would be expected to have a steady TD rate, with further events on a human timescale. When $\gamma$ is larger, or it varies significantly between galaxies, other signatures will likely identify TDs that occurred in binaries. These include possible spectroscopic signatures, morphology, or kinematics indicating a recent major merger or interruption of the TD flare on a binary orbital timescale (Liu et al. 2009).

An ancillary conclusion is that, in our fiducial model, approximately $3 \%$ of all TDs occur in hardening binaries. Therefore, all systems containing a TD are potential binary hosts and should be monitored, either for a second TD or for other evidence of an SMBH binary.

Stone \& Loeb (2011) suggested using multiple TDs due to the merged black holes recoil to identify host galaxies of SMBH mergers observed by LISA. Their period of enhancement is short enough to not affect our conclusions. Also, since LISA will indicate where on the sky $\left(\approx 1 \mathrm{deg}^{2}\right)$ the merger takes place, the high rates discussed here do not affect their conclusions. 
The TD rates are largely determined by the number of stars in the central regions of the galaxy, which, in turn, depends on the cusp profile and the size of the cusp. In this sense, multiple TDs are also diagnostic of cusp profiles.

All our numbers scale by the uncertain detection volume, which could be significantly higher than we have conservatively assumed. Recently, van Velzen et al. (2010) found two candidate disruptions with absolute $g$-band magnitudes -20.3 and -18.3 . If the event with magnitude -20.3 was representative of the higher black hole mass disruptions where our binary-induced disruptions typically occur, then LSST could detect disruptions of this type to $z \sim 0.7$, increasing our predicted rates by approximately an order of magnitude.

We gratefully acknowledge useful discussions with Sterl Phinney, Annika Peter, and Andrew Benson. We also thank David Merritt for providing his fits to ACS Virgo data and Marta Volonteri for calculating the SMBH merger rates. Support for this work was provided by NASA BEFS grant NNX-07AH06G.

\section{REFERENCES}

Aller, M. C., \& Richstone, D. 2002, AJ, 124, 3035

Binney, J., \& Tremaine, S. 2008, Galactic Dynamics (2nd ed.; Princeton, NJ: Princeton Univ. Press)
Chen, X., Madau, P., Sesana, A., \& Liu, F. K. 2009, ApJ, 697, L149

Chen, X., Sesana, A., Madau, P., \& Liu, F. 2011, ApJ, 729, 13

Côté, P., Blakeslee, J., Ferrarese, L., et al. 2004, ApJS, 153, 223

Dehnen, W. 1993, MNRAS, 265, 250

Donley, J. L., Brandt, W. N., Eracleous, M., \& Boller, T. 2002, AJ, 124, 1308

Evans, C. R., \& Kochanek, C. S. 1989, ApJ, 346, L13

Gezari, S. 2010, in IAU Symp. 267, Co-Evolution of Central Black Holes and Galaxies, ed. B. M. Peterson, R. S. Somerville, \& T. Storchi-Bergmann (Cambridge: Cambridge Univ. Press), 319

Gezari, S., Basa, S., Martin, D. C., et al. 2008, ApJ, 676, 944

Ivanov, P. B., Polnarev, A. G., \& Saha, P. 2005, MNRAS, 358, 1361

Liu, F. K., Li, S., \& Chen, X. 2009, ApJ, 706, L133

Lodato, G., \& Rossi, E. M. 2011, MNRAS, 410, 359

Magorrian, J., \& Tremaine, S. 1999, MNRAS, 309, 447

Merritt, D., Schnittman, J. D., \& Komossa, S. 2009, ApJ, 699, 1690

Peter, A. H. G. 2009, Phys. Rev. D, 79, 103531

Phinney, E. S. 1989, in IAU Symp. 136, The Center of the Galaxy, ed. M. Morris (Dordrecht: Kluwer), 543

Preto, M., \& Tremaine, S. 1999, AJ, 118, 2532

Rees, M. J. 1988, Nature, 333, 523

Sesana, A., Haardt, F., \& Madau, P. 2008, ApJ, 686, 432

Stewart, K. R., Bullock, J. S., Barton, E. J., \& Wechsler, R. H. 2009, ApJ, 702, 1005

Stone, N., \& Loeb, A. 2011, MNRAS, 412, 75

Strubbe, L. E., \& Quataert, E. 2009, MNRAS, 400, 2070

Tremaine, S., Richstone, D. O., Byun, Y.-I., et al. 1994, AJ, 107, 634

Ulmer, A. 1999, ApJ, 514, 180

van Velzen, S., Farrar, G. R., Gezari, S., et al. 2010, arXiv:1009.1627

Volonteri, M., Miller, J. M., \& Dotti, M. 2009, ApJ, 703, L86

Wang, J., \& Merritt, D. 2004, ApJ, 600, 149 